\title{
The Awareness of Venous Thromboembolism and Its Prophylaxis: A Survey Study
}

\author{
(D) Nagihan Duran Yakar, (D) Tarkan Mıngır, (D) Nuri Yakar, (D) Cansu Kılınç Berktaş, (D) Namigar Turgut \\ Istanbul Okmeydanı Training and Research Hospital, Clinic of Anesthesiology and Reanimation, Istanbul, Turkey
}

\section{Abstract}

Objective: The purpose of this questionnaire study was to present the results of venous thromboembolism (VTE) questionnaire and compare the data with the literature by having information about awareness level of VTE in anesthesiology and reanimation specialists and branch surgeons and prophylaxis protocols being performed by them.

Methods: A printed questionnaire consisting of seven questions was used for gathering information from the physicians from anesthesiology and reanimation and relevant surgery clinics. The data were given as percentage in statistical analysis.

Results: One hundred and twenty-three participants were included in the study. According to the study results, the participants stated that VTE was a serious cause of mortality; risk factors were questioned in their clinics; they did not use a questionnaire for risk factor and they did not use a risk factor scoring system in risk factor questioning $(84 \%, 89.1 \%, 10.9 \%, 79 \%$, respectively). Eighty-five percent of the participants seemed to perform VTE prophylaxis preoperatively and $76.9 \%$ of them preferred to design both pharmacological and mechanical prophylaxis (MP) together. The second most common choice was that they used MP alone (14.6\%). Seven point three percent of the participants reported that they gave pharmacological prophylaxis alone and $1.2 \%$ of the participants stated that they did not plan any of the prophylaxis methods. The participants used low molecular weight heparin, unfractionated heparin and acetylsalicylic acid among pharmacological methods (70.7\%, $8 \%, 7 \%$, respectively).

Conclusion: Based on data obtained from this study, there is a need for a national risk assessment and thromboprophylaxis policy. Implementation of a change in practice will have the potential to prevent or reduce morbidity and mortality associated with hospital-acquired thrombosis.

Keywords: Prophylaxis, venous thromboembolism, risk

\section{INTRODUCTION}

Venous thromboembolism (VTE) is a spectrum of disease comprising deep vein thrombosis (DVT) and pulmonary embolism (PE). Being a silent disease, VTE is the third leading cause of mortality, followed by heart attack and stroke with a common mechanism of cardiovascular diseases. VTE is also responsible for one in four deaths worldwide (1,2). VTE, which is the number one cause of preventable deaths among hospitalized patients, has been one of the well-known risks of surgery for many years. The most effective and inexpensive method to reduce the mortality and morbidity rates due to VTE is prophylaxis. In fact, thromboprophylaxis (TP) begins with appropriate positioning of the patient on the operating table $(3,4)$. Today, even among health professionals working in this field, it is considered that there is little awareness about its conditions, risks, symptoms and preventive applications. The purpose of this survey study was to present the results of VTE questionnaire and to compare the data with the literature by acquiring information about awareness level of VTE in anesthesiology and reanimation specialists and branch surgeons and prophylaxis protocols being performed by them.

\section{METHODS}

With the permission of local ethics committee of İstanbul Okmeydanı Training and Research Hospital (17.01.2017/588), a printed questionnaire consisting of seven questions was used for gathering information from the anesthesiology and reanimation clinic and relevant surgery clinics (general surgery, plastic surgery, ear-nose-throat, urology, orthopedics, neurosurgery). 


\section{Data Collection}

Anesthesiology and reanimation specialists and physicians from other surgery clinics that participated in the questionnaire study were determined as the sample size and the study aimed to include at least 100 health workers. Anesthesiology and reanimation specialists and physicians from other surgery clinics who agreed to participate in the study were included, and those who did not agree to participate were excluded.

\section{Statistical Analysis}

Demographic data, age, gender and years of professional experience were recorded. Questions about awareness, risk factors, scoring systems, time and methods of prophylaxis and pharmacological prophylaxis were directed to the participants. The data were given as percentages (\%).

\section{RESULTS}

Participants ( $n=123$ ) were selected from physicians and critical care nurses (84.2\% and $15.8 \%$ of participants, respectively), including both men and women (67\% and $33 \%$, respectively). The age of the participants ranged from 25 to 54 years (mean age $=36.6$ years). According to the study results, the

Table 1. Characteristics of patients and questionnaire study; questions and answers

\begin{tabular}{|l|l|l|}
\hline & $\mathrm{n}=123$ & Mean \\
\hline Female & $33 \%$ & \\
\hline Male & $67 \%$ & \\
\hline Age, years & $25-54$ & 36.6 \\
\hline Answers to questions & $84 \%$ \\
\hline Yes, VTE is a severe condition & $16 \%$ \\
\hline $\begin{array}{l}\text { No, VTE is not a serious cause of mortality that } \\
\text { much }\end{array}$ & \multicolumn{2}{|l|}{} \\
\hline A & & \\
\hline
\end{tabular}

Are the risk factors for venous thromboembolism questioned in your clinic?

\begin{tabular}{|l|l|}
\hline Yes & $89.1 \%$ \\
\hline No & $10.9 \%$ \\
\hline
\end{tabular}

Which scoring system do you use/prefer in questioning about risk factors for venous thromboembolism?

\begin{tabular}{|l|l|}
\hline We use Caprini scoring system & $21 \%$ \\
\hline None & $79 \%$ \\
\hline
\end{tabular}

What is your priority choice for a patient whom you decide using venous thromboembolism prophylaxis?

\begin{tabular}{|l|l|}
\hline Pharmacological prophylaxis & $7.3 \%$ \\
\hline Mechanical prophylaxis & $14.6 \%$ \\
\hline Pharmacological + mechanical prophylaxis & $76.9 \%$ \\
\hline Neither & $1.2 \%$ \\
\hline
\end{tabular}

participants stated that VTE was a serious cause of mortality; risk factors were questioned in their clinics; and they did not use a questionnaire for risk factors $(84 \%, 89.1 \%$ and $10.9 \%$ of the participants, respectively) (Table 1). The most common risk factors encountered by surgeons were acute risk factors; $39 \%$ of the surgeons accepted all risk factors as acute risk factors, while $61 \%$ of the surgeons accepted acute risk factors as the following: (a) frequent hospitalization (27\%), (b) frequent chemotherapy administration, (c) frequent administration of estrogen, recently started, (d) frequent intravenous catheterization and (e) frequent immobilization. The acute "triggering" risk factors selected by the participants to use prophylaxis are shown in Table 2. Seventy-nine percent of the participants reported that they did not use a scoring system for risk factor questioning; $85 \%$ of the participants seemed to start using VTE prophylaxis preoperatively and $76.9 \%$ of them preferred to arrange both pharmacological and mechanical prophylaxis together. The second most common use was mechanical prophylaxis alone (14.6\%). Seven point three percent of the participants reported that they gave pharmacological prophylaxis alone and $1.2 \%$ of the participants stated that they did not plan any prophylaxis methods. With respect to the DVT prophylaxis, the participants used low molecular weight heparin, unfractionated heparin and acetylsalicylic acid among pharmacological methods $(70.7 \%, 8 \%$ and $7 \%$, respectively).

\section{DISCUSSION}

With the increasing levels of development of countries, morbidity and mortality due to chronic diseases occur more than contagious diseases. Worldwide deaths due to ischemic heart disease and stroke have increased by $35 \%$ and $25 \%$, respectively, since the 90s (5). VTE substantially contributes to the worldwide disease burden. Although VTE-related morbidity and mortality are considerably preventable, global controls reveal a lack of systemic TP use in patients with medium and high risk of VTE (2).

Table 2. Acute "triggering" risk factors of venous thromboembolism

\begin{tabular}{|l|}
\hline a. Hospitalization \\
\hline b. Surgical intervention \\
\hline c. Lower extremity/pelvic trauma or fracture \\
\hline d. Prolonged travel \\
\hline e. Estrogen therapy starting a short time ago \\
\hline f. Intravenous catheterization \\
\hline g. Chemotherapy \\
\hline h. Immobility \\
\hline i. Pregnancy-postpartum period \\
\hline
\end{tabular}


Cohen et al. (6) described thromboembolism as the "single biggest killer of pregnant women" in their study and in the guidebooks of Royal College of Obstetrics and Gynecologists. Again, DVT occurs in $45 \%$ to $51 \%$ of patients undergoing orthopedic surgery, unless TP is used. The International Society on Thrombosis and Haemostasis has declared $13^{\text {th }}$ October as "World Thrombosis Day" starting in 2014 in order to increase the global awareness of thrombosis. One of the key activities of that day was to conduct a global survey for measuring global public awareness about thrombosis, specifically VTE, thus evaluating the success of the program through follow-up questionnaires. This survey was designed for collecting data about the degree of awareness on VTE, the extent of recognition of the signs and symptoms of DVT and PE. The aim was to share the general information with the public to raise awareness about VTE and enable patients to engage in discussions with their own surgeons and healthcare providers about their individual risk of VTE and their need for TP. The survey was performed in nine representative countries and included respondents of different ages to assess the differences in awareness between generations. General awareness related to VTE was low, in spite of 7233 respondents. The awareness was $19 \%$ for PE, while it was $28 \%$ for DVT. The rate of awareness of underlying causes of DVT was moderately high, additionally, only $45 \%$ of respondents were aware that many blood clots can be prevented, nevertheless, awareness of VTE was low relative to prostate cancer and breast cancer (7). In our country, Reliance platform was constituted and treatment guides were prepared in order to increase the awareness of VTE on the basis of physicians and also society, to educate physicians and allied health personnel about VTE prophylaxis and treatment, to prevent DVT and $\mathrm{PE}$, thus decreasing the morbidity and mortality caused by them and, to contribute for constitution of a national guide about VTE prophylaxis, diagnosis and treatment (4).

VTE is recognized internationally as a serious health problem. VTE is associated with mortality and morbidity in hospitalized medical patients and imposes a huge economic burden for health service. Although it is well established in the literature that active implementation of a mandatory risk assessment tool and an evidence-based TP policy decreases the incidence of hospital associated thrombosis (HAT), only $26 \%(n=8 / 31)$ of hospitals in Ireland was found to have a local implemented TP policy in a national survey distributed to 40 hospitals throughout Ireland in order to examine the utilization of a VTE risk assessment tool and TP policy. Only six of these eight hospitals had a risk assessment tool in conjunction with the TP policy. Based on data obtained from this survey, a need for a national risk assessment and TP policy in Ireland was reported (8). The results of our study were also similar; $89.1 \%$ of the participants reported that they questioned the risk factors in their clinics, but $79 \%$ of them reported that they did not use a scoring system. It was also valid for us. In a study of Oh et al. (9) that was conducted among Korean nurses, only $9.3 \%$ of the nurses reported having received in service VTE education from their hospital. The findings showed that, beyond prevention practices of VTE, the nurses demonstrated a lack of knowledge about VTE and their self-efficacy level was not highly rated. A training focused on prevention of VTE and risk assessment should be considered as a part of continuing education of nurses. In our study, the most frequent risk factors encountered by the surgeons were acute risk factors; $39 \%$ of the surgeons accepted all risk factors as acute, while $61 \%$ of the surgeons accepted acute risk factors as the following: (a) frequent hospitalization (27\%), (b) frequent chemotherapy administration, (c) frequent administration of estrogen, recently started, (d) frequent intravenous catheterization and (e) frequent immobilization. Seventy nine percent of the participants reported that they did not use a scoring system for risk factor questioning. This indicates that education/training courses on VTE for all healthcare workers should also be arranged in our country. Similar to our study, in a study conducted by Özkaya et al. (10) with plastic and reconstructive surgeons, 36\% of the participants reported the time of prophylaxis initiation as preoperative, and many of them reported the time as one day before the operation. Twenty nine percent of the participants reported that prophylaxis was initiated during operation and that the perioperative administration was implemented almost immediately at the beginning of the operation. It is obvious that significant progress has been achieved in recent years, since this rate was $85 \%$ in our study. Only $10 \%$ of the participants reported using prophylaxis during operation. In their study performed in 292 hospitalized adult patients, Ikama et al. (11) identified risk factors such as age and long-term immobilization in order to identify patients at risk of VTE and evaluate the use of preventive measures. They found that $79 \%$ of the surgery candidates received VTE prevention measures, pharmacological prevention was used in $57.4 \%$ of them, mechanical prevention in $19.5 \%$, and the two types of prevention in $23.1 \%$ of them. In our study, $85 \%$ of the participants seemed to start using VTE prophylaxis preoperatively and $76.9 \%$ of them preferred to use both pharmacological and mechanical prophylaxis together. The second most common form of use was mechanical prophylaxis alone (14.6\%). Seven point three percent of the participants stated that they gave pharmacological prophylaxis alone and $1.2 \%$ of the participants stated that they did not plan to implement any of the prophylaxis methods. 


\section{CONCLUSION}

Nevertheless, these data indicate that certain programs should be arranged for identifying the patients at risk and taking preventive measures besides increasing awareness. Based on the data obtained from this study, there is a need for a national risk assessment and TP policy. Implementation of a change in practice will have the potential of preventing or reducing morbidity and mortality associated with HAT.

\section{Ethics}

Ethics Committee Approval: Ethics committee approval was received for this study from the Local Ethics Committee of Okmeydanı Training and Research Hospital (approvel number: 17.01.2017/588).
Informed Consent: A survey study.

Peer-review: External and internal peer-reviewed.

\section{Authorship Contributions}

Surgical and Medical Practices: N.D.Y., T.M., N.Y., C.K.B., N.T., Concept: N.D.Y., N.T., Design: N.D.Y., T.M., N.Y., C.K.B., N.T.,Data Collection or Processing: N.D.Y., T.M., N.Y., C.K.B., Analysis or Interpretation: N.D.Y., N.Y., Literature Search: N.D.Y., T.M., N.Y., Writing: N.D.Y., T.M., N.T.

Conflict of Interest: The authors declare no conflict of interest.

Financial Disclosure: The authors declared that this study received no financial support.

\section{Questionnaire of the Awareness of Venous Thromboembolism and its Prophylaxis}

Age:

Gender:

Occupation:

Number of years in your profession:

Your present working place:

Two or five of every 100 people are predicted to experience venous thromboembolism (VTE) at least one time during their lifetime. Approximately 2 millions/year deep vein thrombosis (DVT) and and 600,000/year pulmonary embolism cases are seen in United States, every year. This number is more than the total number of the people lost because of AIDS (Acquired Immune Deficiency syndrome), breast cancer and traffic accidents.

1. Did you find this information convincing?

a. Yes, VTE is a severe condition

b. No, VTE is not a serious reason of mortality that much

2. Are the risk factors for venous thromboembolism questioned in your clinic?
a. Yes
b. No

3. Which scoring systems do you use/prefer in questioning about risk factors for venous thromboembolism?

None

We use scoring system

4. According to you, which of the following is/are not one of the acute "triggering" risk factors of venous thromboembolism?

a. Hospitalization b. Surgical intervention

c. Lower extremity/pelvic trauma or fracture

d. Prolonged travel

e. Estrogen therapy starting a short time ago

f. Intravenous catheterization

g. Chemotherapy

h. Immobility

i. Pregnancy-postpartum period

5. According to you, what should be the timing for using venous thromboembolism prophylaxis in a patient?
a. Preoperative
b. At operation
c. Immediately after operation
d. Postoperative at the end of $24^{\text {th }}$ hour
e. No idea

7. What is your priority choice for a patient whom you decide using venous thromboembolism prophylaxis?
a. Pharmacological prophylaxis
b. Mechanical prophylaxis
C. Pharmacological + mechanical prophylaxis
d. Neither

8. What does pharmacological prophylaxis mean for you? 


\section{REFERENCES}

1. Bartlett MK, Zhang Y, Kreidler N, Sun S, Ardy R, Cao K, Sack L. Global analysis of plasticity in turgor loss point, a key drought tolerance trait. Ecol Lett 2014;17:1580-90

2. Raskob GE, Silverstein R, Bratzler DW, Heit JA, White RH. Surveillance for deep vein thrombosis and pulmonary embolism: recommendations from a national workshop. Am J Prev Med 2010;38:S502-9.

3. Turpie AG, Leizorovicz A. Prevention of venous thromboembolism in medically ill patients: a clinical update. Postgrad Med J 2006;82:806-9.

4. ULUSAL VENÖZ TROMBOEMBOLIZM PROFILAKSI VE TEDAVI KILAVUZU 2010 Editörler Prof. Dr. Muzaffer Demir Prof. Dr. BülentErdemli Prof. Dr. Mehmet Kurtoğlu Prof. Dr. GülÖngenYayınlayan cortex iletişim hizmetleri a.ș manolyalı sokak no: 22 Levent, İstanbul ISBN 978-605-5720-05-6 Basım Diasan Basım Form Matbaacılık San. Tic. A.Ş BasımYılı 2010

5. Lozano R, Naghavi M, Foreman K, Lim S, Shibuya K, Aboyans V, et al. Global and regional mortality from 235 causes of death for 20 age groups in 1990 and 2010: a systematic analysis for the Global Burden of Disease Study 2010. Lancet 2012;380:2095-128.

6. Cohen A, Botteman M, Stephens J, Ewing M, Collier P, Pashos C, et al. Cost efectiveness of two anticoagulants for the prophylaxis of DVT and subsequent long-term complications (PTS and recurrent VTE) in total hip replacement surgery in the United Kingdom. Poster Presentation. XVIIIth Congress of the International Society on Thrombosis and Haemostasis, Paris, France. ThrombHaemost 2001

7. Wendelboe AM, McCumber M, Hylek EM, Buller H, Weitz JI, Raskob G; ISTH Steering Committee for World Thrombosis Day. Global public awareness of venous thromboembolism. J Thromb Haemost 2015;13:1365-71.

8. Khan MI, O'Leary C, Silvari V, O'Brien A, O'Connor M, Duggan C, et al. Venous Thromboembolism - Risk Assessment Tool and Thromboprophylaxis Policy: A National Survey. Ir Med J 2017;110:499.

9. Oh H, Boo S, Lee JA. Clinical nurses' knowledge and practice of venous thromboembolism risk assessment and prevention in South Korea: a crosssectional survey. J Clin Nurs 2017;26:427-35.

10. Özkaya Ö, Öztürk MB, Egemen 0 , Öreroğlu AR, Üsçetin I, Tasasız K, et al. How Much Do Know About The Venous Thromboembolism? The Approch Of Turkish Plastic Surgeons To The Venous Thromboembolism Prophlaxis And Preferred Methods in Prophylaxis: A Survey Study. Turk Plast Surg 2013;21.

11. Ikama SM, Nkoua MF, Gombet TR, Makani J, Mongo-Ngamami S, OndzeKafata LI, et al. Evaluation of venous thromboembolism risk and prophylaxis among hospitalized patients in Brazzaville. J Mal Vasc 2016;41:182-7. 